\title{
Nucleophilicity Index Based on Atomic Natural Orbitals
}

\author{
Dariusz W. Szczepanik and Janusz Mrozek \\ Department of Computational Methods in Chemistry, Faculty of Chemistry, Jagiellonian University, \\ St. Ingardena 3, 30-060 Cracow, Poland \\ Correspondence should be addressed to Dariusz W. Szczepanik; szczepad@chemia.uj.edu.pl
}

Received 30 May 2013; Accepted 21 August 2013

Academic Editor: K. R. S. Chandrakumar

Copyright (C) 2013 D. W. Szczepanik and J. Mrozek. This is an open access article distributed under the Creative Commons Attribution License, which permits unrestricted use, distribution, and reproduction in any medium, provided the original work is properly cited.

\begin{abstract}
A simple method of evaluating a semilocal (regional) nucleophilicity is introduced. The concept involves use of the natural orbitals for atomic populations to identify the most "reactive population" of electrons on particular atom in molecule. The results of test calculations considering the regioselectivity problem in electrophilic aromatic substitution to the benzene derivatives are presented and briefly discussed.
\end{abstract}

\section{Introduction}

There is a wide variety of reactivity predictors for electrontransfer-controlled reactions among which the Fukui function, originating from the density functional theory (DFT), is one of the most fundamental ones [1,2]. Within the orbitalbased calculations one of the simplest methods to interpret and implement is the condensed Fukui index [3-6] (a coarsegrained atom-by-atom representation of the Fukui function) which can be determined from electron population analysis. There are many arguments $[7,8]$ in favor of the condensed Fukui index based on the Hirshfeld's populational scheme [9]; however, other population analysis methods, including the Mulliken's [10-13] and Löwdin's [14] as well as the NPA schemes [15] are also in common use (e.g., [16-18]).

In this paper we briefly introduce and test a new simple method of evaluating the relative (with respect to particular atom in two "homologous" molecules) reactivity of nucleophiles, based on the natural orbitals for atomic population of electrons [19] and involving their energies as well as occupation numbers. The method is related to the Fukui function concept and its approximation within the framework of Frontier Molecular Orbital (FMO) theory [20-22]. In this work we take into consideration the well-known problem (somewhat trivial but illustrative) of regioselectivity prediction in the electrophilic aromatic substitution to the following benzene derivatives: fluorobenzene $\left(\mathrm{C}_{6} \mathrm{H}_{5} \mathrm{~F}\right)$, aniline
$\left(\mathrm{C}_{6} \mathrm{H}_{5} \mathrm{NH}_{2}\right)$, phenol $\left(\mathrm{C}_{6} \mathrm{H}_{5} \mathrm{OH}\right)$, nitrobenzene $\left(\mathrm{C}_{6} \mathrm{H}_{5} \mathrm{NO}_{2}\right)$, benzoic acid $\left(\mathrm{C}_{6} \mathrm{H}_{5} \mathrm{COOH}\right)$, and benzaldehyde $\left(\mathrm{C}_{6} \mathrm{H}_{5} \mathrm{CHO}\right)$.

In general, functional groups $-\mathrm{OH}$ and $-\mathrm{NH}_{2}$ are classified as electron donating and strongly activating in the electrophilic substitution reactions while functional groups $-\mathrm{NO}_{2},-\mathrm{COOH}$, and $\mathrm{CHO}$ remove electron density from the benzene ring and thus strongly deactivate the molecule. Functional groups from the former class tend to be ortho/para directing while those from the latter one direct electrophiles to attack the benzene molecule at the meta position. In fluorobenzene (likewise in other benzene halides) the benzene ring is weakly deactivated due to inductive withdrawal of electrons by electronegative atom F. However, the resonance donation of nonbonding electrons of fluorine atom to the benzene ring causes that the most preferable positions of electrophilic attack are ortho and para.

\section{Method Details}

Let us assume the closed-shell molecular system with $N$ electrons doubly occupying $n$ lowest molecular orbitals $\left|\varphi^{\circ}\right\rangle=$ $\left\{\left|\varphi_{1}\right\rangle, \ldots,\left|\varphi_{n}\right\rangle \equiv\left|\varphi^{*}\right\rangle\right\}$, generated as linear combinations of orthogonalized atomic orbitals (OAO) $|\chi\rangle$,

$$
\left|\varphi_{i}\right\rangle=\sum_{\alpha}\left|\chi_{\alpha}\right\rangle\left\langle\chi_{\alpha} \mid \varphi_{i}\right\rangle=\sum_{\alpha}\left|\chi_{\alpha}\right\rangle C_{\alpha, i}
$$


or in matrix form,

$$
\left|\varphi^{o}\right\rangle=|\chi\rangle\left\langle\chi \mid \varphi^{o}\right\rangle=|\chi\rangle \mathbf{C}^{o},
$$

where the rectangular matrix $\mathbf{C}^{o}$ groups the relevant LCAO $\mathrm{MO}$ expansion coefficients. It follows directly from the superposition principle of quantum mechanics that the conditional probability of "finding electron" from $i$ th occupied molecular orbital on $\alpha$ th atomic orbital reads

$$
P_{i}(\alpha)=\left\langle\chi_{\alpha} \mid \varphi_{i}\right\rangle\left\langle\varphi_{i} \mid \chi_{\alpha}\right\rangle=\left\langle\chi_{\alpha}\left|\widehat{P}_{i}\right| \chi_{\alpha}\right\rangle=\left|C_{\alpha, i}\right|^{2} .
$$

Hence, the LCAO MO expansion coefficient $C_{\alpha, i}$ can be regarded as the conditional-probability amplitude. Alternatively, one can relate $C_{\alpha, i}$ to the amplitude of the probability of "finding electron" from $\alpha$ th atomic orbital on $i$ th canonical molecular orbital. Then

$$
P_{\alpha}(i)=\left\langle\varphi_{i} \mid \chi_{\alpha}\right\rangle\left\langle\chi_{\alpha} \mid \varphi_{i}\right\rangle=\left\langle\varphi_{i}\left|\widehat{P}_{\alpha}\right| \varphi_{i}\right\rangle=\left|C_{\alpha, i}\right|^{2} .
$$

Similarly, by replacing the operator $\widehat{P}_{\alpha}$ with the operator $\widehat{P}_{X}$ projecting onto the subspace of all OAOs centered on atom $X$, we get the conditional probability of the event that the electron from electron population on atom $X$ can be ascribed to $i$ th molecular orbital,

$$
\begin{aligned}
P_{X}(i) & =\mathscr{N}_{X}\left\langle\varphi_{i}\left|\widehat{P}_{X}\right| \varphi_{i}\right\rangle=\mathscr{N}_{X} \sum_{\alpha}^{X}\left\langle\varphi_{i}\left|\widehat{P}_{\alpha}\right| \varphi_{i}\right\rangle \\
& =\mathscr{N}_{X} \sum_{\alpha}^{X}\left|C_{\alpha, i}\right|^{2},
\end{aligned}
$$

where normalization constant reads

$$
\mathcal{N}_{X}=\left[\sum_{i}^{n} P_{X}(i)\right]^{-1}=\left[\sum_{i}^{n} \sum_{\alpha}^{X}\left|C_{\alpha, i}\right|^{2}\right]^{-1} .
$$

The corresponding $n \times n$ matrix $\mathbf{P}_{X}\left(\varphi^{\circ}, \varphi^{\circ}\right)$ of elements representing projections of $j$ th occupied MO onto $i$ th occupied MO through the subspace of AOs assigned to atom $X$ [23],

$$
\begin{aligned}
\mathbf{P}_{X} & \left(\boldsymbol{\varphi}^{o}, \varphi^{o}\right) \\
& =\left\{P_{X}(i, j)=\mathcal{N}_{X} \sum_{\alpha}^{X}\left\langle\varphi_{i}\left|\widehat{P}_{\alpha}\right| \varphi_{j}\right\rangle=\mathcal{N}_{X} \sum_{\alpha}^{X} C_{\alpha, i} C_{\alpha, j}\right\},
\end{aligned}
$$

is obviously not diagonal. It follows directly from orthonormality of molecular orbitals that regarding the whole (molecular) electron population $N$,

$$
\begin{aligned}
\mathbf{P}\left(\varphi^{o}, \varphi^{o}\right) & =\sum_{X} \mathbf{P}_{X}\left(\varphi^{o}, \varphi^{o}\right) \\
& =\mathcal{N}\left\langle\varphi^{o}\left|\sum_{X} \widehat{P}_{X}\right| \boldsymbol{\varphi}^{o}\right\rangle=\mathcal{N}\left\langle\varphi^{o} \mid \varphi^{o}\right\rangle=\frac{1}{n} \mathbf{1}_{n} .
\end{aligned}
$$

The representation of orbitals $\left|\boldsymbol{\theta}_{X}\right\rangle$,

$$
\left|\boldsymbol{\theta}_{X}\right\rangle=\left|\boldsymbol{\varphi}^{o}\right\rangle\left\langle\boldsymbol{\varphi}^{o} \mid \boldsymbol{\theta}_{X}\right\rangle=\left|\boldsymbol{\varphi}^{o}\right\rangle \mathbf{C}_{X}^{\theta}
$$

in which off-diagonal elements of matrix (7) are zeroes, $\left\{P_{X}(i, j)=\delta_{i, j}\right\}$; one can straightforwardly obtain from diagonalization of $\mathbf{P}_{X}\left(\varphi^{o}, \varphi^{o}\right)$

$$
\mathbf{P}_{X}\left(\varphi^{o}, \varphi^{o}\right)=\mathbf{C}_{X}^{\theta} \mathbf{p}_{X}^{\theta} \mathbf{C}_{X}^{\theta \dagger} .
$$

The diagonal matrix of electron probabilities $\mathbf{p}_{X}^{\theta}$ can be used to calculate the electron populations $\mathbf{n}_{X}^{\theta}$ of the corresponding natural orbitals for electron population of atom $X,\left|\boldsymbol{\theta}_{X}\right\rangle$,

$$
\mathbf{n}_{X}^{\theta}=N_{X} \mathbf{p}_{X}^{\theta}, \quad \text { where } N_{X}=2 \sum_{i}^{n} \sum_{\alpha}^{X}\left|C_{\alpha, i}\right|^{2}
$$

stands for the electron population on atom $X$. The corresponding matrix of the 1-electron orbital "energies" $\varepsilon_{X}^{\theta}$ (i.e., expectation values of $\widehat{F}^{\varphi}$ in $\left.\left|\boldsymbol{\theta}_{X}\right\rangle\right)$ can be determined as follows:

$$
\boldsymbol{\varepsilon}_{X}^{\theta}=\left\langle\boldsymbol{\theta}_{X}\left|\widehat{F}^{\varphi}\right| \boldsymbol{\theta}_{X}\right\rangle=\mathbf{C}_{X}^{\theta \dagger}\left\langle\boldsymbol{\varphi}^{o}\left|\widehat{F}^{\varphi}\right| \boldsymbol{\varphi}^{o}\right\rangle \mathbf{C}_{X}^{\theta}=\mathbf{C}_{X}^{\theta \dagger} \boldsymbol{\varepsilon}^{o} \mathbf{C}_{X}^{\theta} .
$$

Here, $\widehat{F}^{\varphi}$ is the Fock operator, and diagonal matrix $\varepsilon^{o}$ collects orbital energies of occupied canonical MOs. Since natural orbitals $\left|\boldsymbol{\theta}_{X}\right\rangle$ are not the eigenvectors of $\widehat{F}^{\varphi}$, the matrix of orbital energies (12) is not diagonal. Orbital energies of both frontier canonical molecular orbitals, HOMO $\left(\varepsilon^{*} \equiv \varepsilon^{\mathrm{HOMO}} \equiv \varepsilon_{n, n},\right)$ and LUMO $\left(\varepsilon^{\mathrm{LUMO}} \equiv \varepsilon_{n+1, n+1},\right)$, are known to be useful qualitative indicators of chemical reactivity. Thus, it is of our special interest to investigate how the expectation values of operator $\widehat{F}^{\varphi}$ within representation of $\left|\boldsymbol{\theta}_{X}\right\rangle$ manage with evaluation of the reactivity of the particular atom $X$. However, due to nonorthogonality of natural-orbital sets for different atoms, only the relative changes of $\boldsymbol{\varepsilon}_{X}^{\theta}$ for the same $X$ in two homologous species should be compared.

One of the standard frontier-orbital treatments of chemical reactivity is the regioselectivity problem, for example, in the electrophilic aromatic substitution to the benzene derivatives. The standard FMO theory analysis of the effect of substituent groups involves the electron populations of the highest occupied molecular orbital (HOMO), whereas within the newly proposed "reverse scenario" we focus on the electron population of particular atom first and then analyze the highest "energies" of occupied atomic NOs.

\section{Numerical Results}

To examine the presented methodology we have used statefunctions calculated at RHF(ROHF for cations)/STO-3G, $\mathrm{RHF}(\mathrm{ROHF}) / 6-31 \mathrm{G}^{*}$ as well as DFT/B3LYP/aug-cc-pVDZ theory levels, using the standard ab initio quantum chemistry package GAMESS [24], for the following benzene derivatives: fluorobenzene, aniline, phenol, nitrobenzene, benzoic acid, and benzaldehyde. The highest energies of occupied natural orbitals and the corresponding electron populations were compared with two standard MO-based atomic descriptors of reactivity: the atomic index of nucleophilicity [25] involving only the highest occupied $\mathrm{MO},\left|\varphi^{*}\right\rangle$,

$$
R_{X}=\frac{\sum_{\alpha}^{X}\left|C_{\alpha, n}\right|^{2}}{\left(1-\varepsilon_{n, n}\right)}=\frac{\sum_{\alpha}^{X}\left|C_{\alpha}^{*}\right|^{2}}{\left(1-\varepsilon^{*}\right)}
$$


TABLE 1: Atomic indices of nucleophilicity (13) and condensed atomic Fukui indices (14) for selected benzene derivatives. For asymmetric species, the position refers to carbon atom with larger electron population. Methods: RHF/ROHF, basis sets: STO-3G, 6-31G* .

\begin{tabular}{|c|c|c|c|c|c|c|c|c|c|}
\hline \multirow{2}{*}{ Molecule } & \multirow{2}{*}{ Position } & \multicolumn{4}{|c|}{ STO-3G } & \multicolumn{4}{|c|}{$6-31 G^{*}$} \\
\hline & & $R_{X}^{\mathscr{M}}$ & $R_{X}^{\mathscr{L}}$ & $F_{X}^{\mathscr{M}}$ & $F_{X}^{\mathscr{L}}$ & $R_{X}^{M}$ & $R_{X}^{\mathscr{L}}$ & $F_{X}^{\mathscr{M}}$ & $F_{X}^{\mathscr{L}}$ \\
\hline \multirow{3}{*}{ Fluorobenzene } & ortho & 0.083 & 0.083 & 0.063 & 0.074 & 0.076 & 0.076 & 0.039 & 0.065 \\
\hline & meta & 0.046 & 0.046 & 0.037 & 0.040 & 0.057 & 0.058 & 0.013 & 0.056 \\
\hline & para & 0.236 & 0.236 & 0.136 & 0.185 & 0.250 & 0.247 & 0.142 & 0.227 \\
\hline \multirow{3}{*}{ Aniline } & ortho & 0.093 & 0.093 & 0.075 & 0.094 & 0.099 & 0.099 & 0.077 & 0.103 \\
\hline & meta & 0.032 & 0.031 & 0.013 & 0.008 & 0.033 & 0.035 & -0.025 & 0.012 \\
\hline & para & 0.205 & 0.205 & 0.104 & 0.140 & 0.218 & 0.214 & 0.137 & 0.207 \\
\hline \multirow{3}{*}{ Phenol } & ortho & 0.103 & 0.103 & 0.084 & 0.104 & 0.104 & 0.103 & 0.065 & 0.096 \\
\hline & meta & 0.026 & 0.026 & 0.017 & 0.012 & 0.030 & 0.033 & -0.017 & 0.021 \\
\hline & para & 0.221 & 0.221 & 0.124 & 0.168 & 0.238 & 0.235 & 0.142 & 0.220 \\
\hline \multirow{3}{*}{ Nitrobenzene } & ortho & 0.000 & 0.000 & 0.036 & 0.039 & 0.180 & 0.179 & 0.034 & 0.038 \\
\hline & meta & 0.000 & 0.000 & 0.020 & 0.020 & 0.182 & 0.180 & -0.004 & 0.023 \\
\hline & para & 0.000 & 0.000 & 0.052 & 0.068 & 0.003 & 0.006 & 0.050 & 0.083 \\
\hline \multirow{3}{*}{ Benzoic Acid } & ortho & 0.050 & 0.050 & 0.042 & 0.047 & 0.143 & 0.142 & 0.038 & 0.047 \\
\hline & meta & 0.065 & 0.065 & 0.017 & 0.015 & 0.220 & 0.217 & -0.002 & 0.022 \\
\hline & para & 0.222 & 0.222 & 0.051 & 0.067 & 0.010 & 0.012 & 0.042 & 0.074 \\
\hline \multirow{3}{*}{ Benzaldehyde } & ortho & 0.051 & 0.051 & 0.050 & 0.057 & 0.017 & 0.019 & 0.046 & 0.058 \\
\hline & meta & 0.069 & 0.069 & 0.016 & 0.014 & 0.226 & 0.223 & -0.008 & 0.016 \\
\hline & para & 0.227 & 0.227 & 0.061 & 0.080 & 0.117 & 0.117 & 0.053 & 0.089 \\
\hline
\end{tabular}

and the Fukui nucleophilic reactivity index [26] involving electron populations on atom $X$ in cation, $N_{X}^{+}$, and neutral molecule, $N_{X}$,

$$
F_{X}=N_{X}-N_{X}^{+}
$$

Both indices were calculated only within basis sets STO-3G and 6-31G*, since larger basis sets (especially those including diffuse functions, e.g., aug-cc-pVDZ) are well known to dramatically lose their "atomic attributeness" of AOs, and, consequently, many population-type descriptors usually assume completely unreasonable values. Two alternative procedures of population analysis were used in calculation of indices (13) and (14): the Mulliken's scheme (superscript $\mathscr{M}$ ) [10-13] and the Löwdin's one (superscript $\mathscr{L}$ ) [14], involving the standard "geometrical" orthogonalization of atomic orbitals.

The results presented in Table 1 clearly indicate that only index $R_{X}$ calculated within extended basis set allows one to correctly predict the position of electrophilic attack in all molecules under consideration. The Fukui nucleophilic reactivity index $F_{X}$ properly copes with ortho/para directing, but it completely fails with respect to meta directing. It is worth notice that indicator $R_{X}$, involving only electron populations and energies of HOMO, is far less sensitive to population analysis method than index $F_{X}$. Furthermore, if one excludes the results for meta directing groups, there is no significant difference between $R_{X}$ calculated within STO-3G and $6-31 \mathrm{G}^{*}$ basis sets.

We performed a similar analysis using the newly proposed method based on natural orbitals $\left|\boldsymbol{\theta}_{X}\right\rangle$. Table 2 collects orbital occupation numbers $n_{X}^{*}$ and the corresponding relative "energetic effects", $\Delta \varepsilon_{X}^{*}$, calculated as a difference between the highest energy of occupied natural orbitals of particular carbon atoms in benzene and its derivative:

$$
\Delta \varepsilon_{X}^{*}=\varepsilon_{X}^{*} \text { (derivative) }-\varepsilon_{X}^{*} \text { (benzene) } .
$$

Even a cursory analysis of occupation numbers in Table 2 allows one to correctly predict the preferential ortho/para or meta directing of substituent groups, regardless of basis sets used in calculations. However, evaluation of energetic effects provides more detailed information about activating/deactivating influence of substituent groups on particular carbon atoms. For example, $-\mathrm{NH}_{2}$ is properly recognized as the best activator, and the most activated carbon atom is in position para (activated for about $11 \mathrm{kcal} \cdot \mathrm{mol}^{-1}$ ), while substituent group $-\mathrm{NO}_{2}$ strongly deactivates the molecule for about $20-25 \mathrm{kcal} \cdot \mathrm{mol}^{-1}$, and the most active population of electrons is then located on carbon atom in position meta. For the majority of cases orbital energies properly predict the position of electrophilic substitution.

In calculations of natural orbitals from Table 2 we used the standard Löwdin's orthogonalization procedure to obtain orthogonal AOs. However, it was of our interest to find how $\triangle \varepsilon_{X}^{*}$ and $n_{X}^{*}$ depend on the orthogonalization procedure. Table 3 contains the same data as Table 2 but is calculated within representation of "physically" orthogonalized atomic orbitals (superscript $\mathscr{P}$ ) [27-29]. At first glance one can observe a small improvement of electron populations from DFT/B3LYP/aug-cc-pVDZ. Also, in contrast to calculations involving "geometrical" orthogonalization, $\Delta \varepsilon_{X}^{* \mathscr{P}}$ 
TABLE 2: Occupation numbers (11) and differences between the highest NO "energies" (15) in [kcal·moL $\left.{ }^{-1}\right]$ for benzene and its derivatives, calculated within representation of "geometrically" orthogonalized atomic orbitals. For asymmetric species, the position refers to carbon atom with larger electron population. Methods: RHF/STO-3G, 6-31G*, and DFT/B3LYP/aug-cc-pVDZ.

\begin{tabular}{|c|c|c|c|c|c|c|c|}
\hline \multirow{2}{*}{ Molecule } & \multirow{2}{*}{ Position } & \multicolumn{2}{|c|}{ STO-3G } & \multicolumn{2}{|c|}{$6-31 G^{*}$} & \multicolumn{2}{|c|}{ Aug-cc-pVDZ } \\
\hline & & $n_{X}^{* \mathscr{L}}$ & $\Delta \varepsilon_{X}^{* \mathscr{L}}$ & $n_{X}^{* \mathscr{L}}$ & $\Delta \varepsilon_{X}^{* \mathscr{L}}$ & $n_{X}^{* \mathscr{L}}$ & $\Delta \varepsilon_{X}^{* \mathscr{L}}$ \\
\hline \multirow{3}{*}{ Fluorobenzene } & ortho & 1.039 & -2.5 & 1.033 & -8.2 & 0.786 & -7.3 \\
\hline & meta & 0.985 & -7.3 & 0.958 & -10.0 & 0.760 & -7.5 \\
\hline & para & 1.020 & -1.3 & 1.013 & -4.2 & 0.778 & -3.7 \\
\hline \multirow{3}{*}{ Aniline } & ortho & 1.057 & 3.8 & 1.073 & 8.2 & 0.803 & 9.0 \\
\hline & meta & 0.983 & -1.4 & 0.949 & -1.6 & 0.760 & 3.6 \\
\hline & para & 1.035 & 6.7 & 1.047 & 10.4 & 0.794 & 11.0 \\
\hline \multirow{3}{*}{ Phenol } & ortho & 1.046 & 5.0 & 1.048 & 3.5 & 0.793 & 3.3 \\
\hline & meta & 0.979 & -3.7 & 0.949 & -4.4 & 0.758 & -0.9 \\
\hline & para & 1.035 & 4.3 & 1.037 & 4.6 & 0.788 & 4.8 \\
\hline \multirow{3}{*}{ Nitrobenzene } & ortho & 0.971 & -23.7 & 0.928 & -27.2 & 0.738 & -23.0 \\
\hline & meta & 0.999 & -19.6 & 0.992 & -20.8 & 0.763 & -19.1 \\
\hline & para & 0.968 & -20.5 & 0.930 & -24.2 & 0.744 & -20.6 \\
\hline \multirow{3}{*}{ Benzoic acid } & ortho & 0.974 & -7.0 & 0.936 & -12.2 & 0.737 & -11.2 \\
\hline & meta & 1.005 & -6.3 & 0.999 & -8.5 & 0.767 & -9.0 \\
\hline & para & 0.978 & -7.5 & 0.940 & -12.0 & 0.748 & -10.9 \\
\hline \multirow{3}{*}{ Benzaldehyde } & ortho & 0.989 & -6.7 & 0.954 & -14.8 & 0.739 & -14.5 \\
\hline & meta & 1.004 & -6.6 & 0.996 & -10.9 & 0.768 & -11.9 \\
\hline & para & 0.984 & -6.3 & 0.943 & -13.1 & 0.746 & -13.3 \\
\hline
\end{tabular}

TABLE 3: Occupation numbers (11) and differences between the highest NO "energies" (15) in $\left[\mathrm{kcal} \cdot \mathrm{mol}^{-1}\right]$ for benzene and its derivatives, calculated within representation of "physically" orthogonalized atomic orbitals. For asymmetric species, the position refers to carbon atom with larger electron population. Methods: RHF/STO-3G, 6-31G* , and DFT/B3LYP/aug-cc-pVDZ.

\begin{tabular}{|c|c|c|c|c|c|c|c|}
\hline \multirow{2}{*}{ Molecule } & \multirow{2}{*}{ Position } & \multicolumn{2}{|c|}{ STO-3G } & \multicolumn{2}{|c|}{$6-31 G^{*}$} & \multicolumn{2}{|c|}{ Aug-cc-pVDZ } \\
\hline & & $n_{X}^{* \mathscr{P}}$ & $\Delta \varepsilon_{X}^{* \mathscr{P}}$ & $n_{X}^{* \mathscr{P}}$ & $\Delta \varepsilon_{X}^{* \mathscr{P}}$ & $n_{X}^{* \mathscr{P}}$ & $\Delta \varepsilon_{X}^{* \mathscr{P}}$ \\
\hline \multirow{3}{*}{ Fluorobenzene } & ortho & 1.035 & -2.8 & 1.059 & -8.7 & 0.984 & -6.2 \\
\hline & meta & 0.981 & -7.3 & 0.961 & -9.9 & 0.984 & -8.3 \\
\hline & para & 1.018 & -1.8 & 1.028 & -5.3 & 1.000 & -4.5 \\
\hline \multirow{3}{*}{ Aniline } & ortho & 1.053 & 5.6 & 1.100 & 5.5 & 1.033 & 6.7 \\
\hline & meta & 0.978 & -1.2 & 0.945 & -1.1 & 0.989 & 2.2 \\
\hline & para & 1.034 & 6.3 & 1.068 & 8.2 & 1.019 & 9.5 \\
\hline \multirow{3}{*}{ Phenol } & ortho & 1.041 & 4.5 & 1.068 & 2.2 & 1.014 & 3.8 \\
\hline & meta & 0.975 & -3.9 & 0.947 & -4.7 & 0.983 & -1.7 \\
\hline & para & 1.033 & 3.8 & 1.056 & 3.5 & 1.016 & 4.3 \\
\hline \multirow{3}{*}{ Nitrobenzene } & ortho & 0.959 & -23.6 & 0.900 & -26.5 & 0.910 & -21.5 \\
\hline & meta & 0.998 & -19.6 & 1.013 & -21.1 & 0.992 & -20.0 \\
\hline & para & 0.963 & -21.1 & 0.927 & -25.5 & 0.945 & -21.8 \\
\hline \multirow{3}{*}{ Benzoic acid } & ortho & 0.967 & -7.3 & 0.920 & -12.3 & 0.945 & -11.3 \\
\hline & meta & 1.007 & -6.3 & 1.023 & -8.6 & 0.997 & -9.4 \\
\hline & para & 0.976 & -7.8 & 0.942 & -12.8 & 0.955 & -11.9 \\
\hline \multirow{3}{*}{ Benzaldehyde } & ortho & 0.986 & -7.0 & 0.959 & -15.0 & 0.959 & -14.7 \\
\hline & meta & 1.004 & -6.5 & 1.016 & -10.8 & 0.997 & -12.2 \\
\hline & para & 0.982 & -6.6 & 0.947 & -13.7 & 0.956 & -14.0 \\
\hline
\end{tabular}

for benzaldehyde in STO-3G basis set correctly predict meta directing of substituent group. In general, however, calculated energies are similar to those from Table 2 (average distances between the respective energies calculated within "geometrically" and "physically" orthogonalized AO representations are: 0.4 (STO-3G), $0.8\left(6-31 \mathrm{G}^{*}\right)$, and 0.9 (aug-cc$\mathrm{pVDZ}$ ) $\left.\left[\mathrm{kcal} \cdot \mathrm{mol}^{-1}\right]\right)$ and thus allow one to draw almost the same conclusions about reactivity of particular carbon atom. 
The energetic effects from Tables 2 and 3 are relatively small (but comparable with differences between stabilization energies of the corresponding Wheland intermediates [30]). For comparison, below we present several simple species ordered with respect to relative nucleophilicities (from weak to very good nucleophiles) and the corresponding energies $\Delta \varepsilon_{X}^{* \mathscr{L}}\left[\mathrm{kcal} \cdot \mathrm{mol}^{-1}\right]$ (of orbitals identified as lone pairs in all cases) calculated at DFT/B3LYP/TZP with model core potential $[31,32]$ :

$$
\begin{aligned}
& \mathrm{CH}_{3} \mathrm{COOH}<\mathrm{H}_{2} \mathrm{O}<\mathrm{NH}_{3}<\mathrm{CH}_{3} \mathrm{O}^{-}<\mathrm{CH}_{3} \mathrm{~S}^{-} \text {, }
\end{aligned}
$$

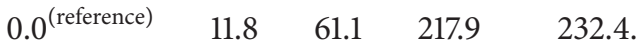

\section{Summary}

In this work we have briefly introduced a simple method of evaluating the relative nucleophilicity in energy scale. The concept involves the use of natural orbitals for atomic population of electrons and their energies (i.e., expectation values of Fock operator) as well as occupation numbers to identify "the most reactive population of electrons" on particular atom (or molecular fragment). Such scenario is directly related to the standard FMO theory treatment involving atomic populations of electrons of the highest occupied molecular orbital (HOMO); in the newly proposed approach we first focus on the electron population of particular atom and then analyze energies of occupied natural orbital. This strategy has been examined on the regioselectivity problem in the electrophilic aromatic substitution to the benzene derivatives. Analysis of the results allows one to draw the conclusion that evaluation and comparison of relative chemical nucleophilicities of atoms in an energy domain are more reliable and advantageous than analyses involving other popular MO-based; this seems to be somehow obvious since, in contradistinction to the majority of condensed atomic indices, energetic descriptors converge systematically to the complete-basis-set limit. It has to be noticed, however, that electron population of the highest occupied natural orbital of a particular atom in molecule is somewhat insensitive to basis set variations and the corresponding energy seems to exhibit the basis set dependence quite consistent with the variational principle.

The presented methodology is still in need of thorough analysis and examination. Also, it is of our special interest to take advantage of the approach based on natural atomic orbitals involving virtual molecular orbitals to evaluate the energetic descriptor of electrophilicity of atoms and molecular fragments.

\section{References}

[1] R. G. Parr and W. T. Yang J, "Density functional approach to the frontier-electron theory of chemical reactivity," Journal of the American Chemical Society, vol. 106, no. 14, pp. 4049-4050, 1984.

[2] P. W. Ayers and M. Levy, "Perspective on 'Density functional approach to the frontier-electron theory of chemical reactivity", Theoretical Chemistry Accounts, vol. 103, no. 3-4, pp. 353-360, 2000.

[3] W. T. Yang and W. J. Mortier, "The use of global and local molecular parameters for the analysis of the gas-phase basicity of amines," Journal of the American Chemical Society, vol. 108, no. 19 , pp. 5708-5711, 1986.

[4] P. Fuentealba, P. Pérez, and R. Contreras, "On the condensed Fukui function," The Journal of Chemical Physics, vol. 113, no. 7, Article ID 2544, 8 pages, 2000.

[5] N. Otero, M. Mandado, and R. A. Mosquera, "Revisiting the calculation of condensed Fukui functions using the quantum theory of atoms in molecules," The Journal of Chemical Physics, vol. 126, no. 23, Article ID 234108, 6 pages, 2007.

[6] P. Bultinck, S. Fias, C. van Alsenoy, P. W. Ayers, and R. CarbóDorca, "Critical thoughts on computing atom condensed Fukui functions," The Journal of Chemical Physics, vol. 127, no. 3, Article ID 034102, 11 pages, 2007.

[7] R. F. Nalewajski and R. G. Parr, "Information theory, atoms in molecules, and molecular similarity," Proceedings of the National Academy of Sciences of the United States of America, vol. 97, no. 16, pp. 8879-8882, 2000.

[8] P. W. Ayers, "Atoms in molecules, an axiomatic approach. I. Maximum transferability," The Journal of Chemical Physics, vol. 113, no. 24, pp. 10886-10898, 2000.

[9] F. L. Hirshfeld, "Bonded-atom fragments for describing molecular charge densities," Theoretica Chimica Acta, vol. 44, no. 2, pp. 129-138, 1977.

[10] R. S. Mulliken, "Electronic population analysis on LCAO-MO molecular wave functions. I," The Journal of Chemical Physics, vol. 23, no. 10, Article ID 1833, 8 pages, 1955.

[11] R. S. Mulliken, "Electronic population analysis on LCAOMO molecular wave functions. II. Overlap populations, bond orders, and covalent bond energies," The Journal of Chemical Physics, vol. 23, no. 10, Article ID 1841, 6 pages, 1955.

[12] R. S. Mulliken, "Electronic population analysis on LCAO-MO molecular wave functions. IV. Bonding and antibonding in LCAO and valence-bond theories," The Journal of Chemical Physics, vol. 23, no. 12, pp. 2343-2346, 1955.

[13] R. S. Mulliken, "Electronic population analysis on LCAOMO molecular wave functions. III. Effects of hybridization on overlap and gross AO populations," The Journal of Chemical Physics, vol. 23, no. 12, Article ID 2338, 5 pages, 1955.

[14] P. O. Löwdin, "On the non-orthogonality problem connected with the use of atomic wave functions in the theory of molecules and crystals," The Journal of Chemical Physics, vol. 18, no. 3, Article ID 365, 11 pages, 1950.

[15] A. E. Reed, R. B. Weinstock, and F. Weinhold, "Natural population analysis," The Journal of Chemical Physics, vol. 83, no. 2, Article ID 735, 12 pages, 1985.

[16] S. Arulmozhiraja and P. Kolandaivel, "Condensed Fukui function: dependency on atomic charges," Molecular Physics, vol. 90, no. 1, pp. 55-62, 1997.

[17] R. K. Roy, K. Hirao, S. Krishnamurty, and S. Pal, "Mulliken population analysis based evaluation of condensed Fukui function indices using fractional molecular charge," The Journal of Chemical Physics, vol. 115, no. 7, pp. 2901-2907, 2001.

[18] F. de Proft, C. van Alsenoy, A. Peeters, W. Langenaeker, and P. Geerlings, "Atomic charges, dipole moments, and Fukui functions using the Hirshfeld partitioning of the electron density," Journal of Computational Chemistry, vol. 23, no. 12, pp. 1198-1209, 2002.

[19] D. Szczepanik and J. Mrozek, "On quadratic bond-order decomposition within molecular orbital space," Journal of Mathematical Chemistry, vol. 51, no. 6, pp. 1619-1633, 2013.

[20] K. Fukui, T. Yonezawa, and H. Shingu, "A molecular orbital theory of reactivity in aromatic hydrocarbons," The Journal of Chemical Physics, vol. 20, no. 4, pp. 722-725, 1952. 
[21] K. Fukui, T. Yonezawa, and C. Nagata, "A free-electron model for discussing reactivity in unsaturated hydrocarbons," The Journal of Chemical Physics, vol. 21, no. 1, pp. 174-176, 1953.

[22] M. Berkowitz, "Density functional approach to frontier controlled reactions," Journal of the American Chemical Society, vol. 109, no. 16, pp. 4823-4825, 1987.

[23] R. F. Nalewajski, D. Szczepanik, and J. Mrozek, "Bond differentiation and orbital decoupling in the orbital-communication theory of the chemical bond," Advances in Quantum Chemistry, vol. 61, pp. 1-48, 2011.

[24] M. W. Schmidt, K. K. Baldridge, J. A. Boatz et al., "General atomic and molecular electronic structure system," Journal of Computational Chemistry, vol. 14, no. 11, pp. 1347-1363, 1993.

[25] R. Franke, Theorecital Drug Design Methods, Elsevier, Amsterdam, The Netherlands, 1984.

[26] J. Melin, P. W. Ayers, and J. V. Ortiz, "Removing electrons can increase the electron density: a computational study of negative fukui functions," The Journal of Physical Chemistry A, vol. 111, no. 40, pp. 10017-10019, 2007.

[27] D. Szczepanik and J. Mrozek, "Symmetrical orthogonalization within linear space of molecular orbitals," Chemical Physics Letters, vol. 512, pp. 157-160, 2012.

[28] D. Szczepanik and J. Mrozek, "Electron population analysis using a reference minimal set of atomic orbitals," Computational and Theoretical Chemistry, vol. 996, pp. 103-109, 2012.

[29] D. Szczepanik and J. Mrozek, "On several alternatives for Löwdin orthogonalization," Computational and Theoretical Chemistry, vol. 1008, pp. 15-19, 2013.

[30] G. W. Wheland, "A quantum mechanical investigation of the orientation of substituants in aromatic molecules," Journal of the American Chemical Society, vol. 64, no. 4, pp. 900-908, 1942.

[31] Y. Sakai, E. Miyoshi, M. Klobukowski, and S. Huzinaga, "Model potentials for main group elements Li through Rn," The Journal of Chemical Physics, vol. 106, no. 19, pp. 8084-8092, 1997.

[32] T. Noro, M. Sekiya, and T. Koga, "Contracted polarization functions for the atoms helium through neon," Theoretical Chemistry Accounts, vol. 98, no. 1, pp. 25-32, 1997. 

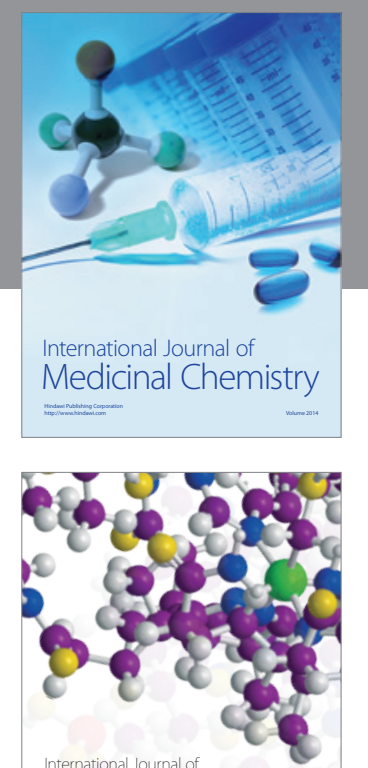

\section{Carbohydrate} Chemistry

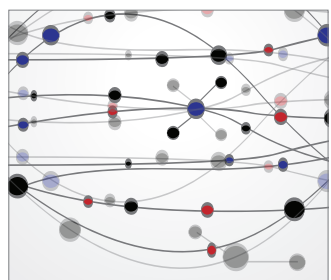

The Scientific World Journal
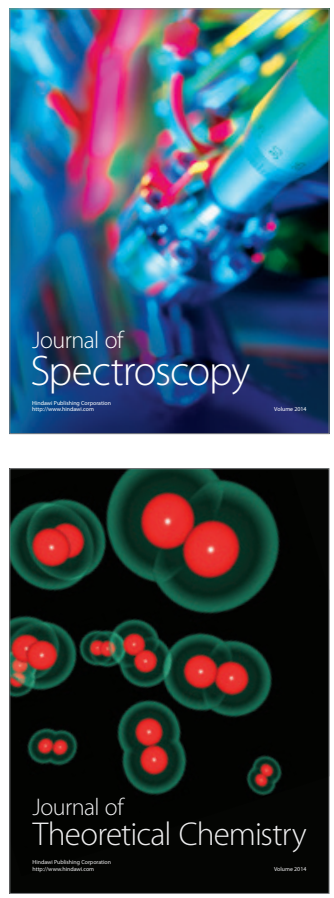
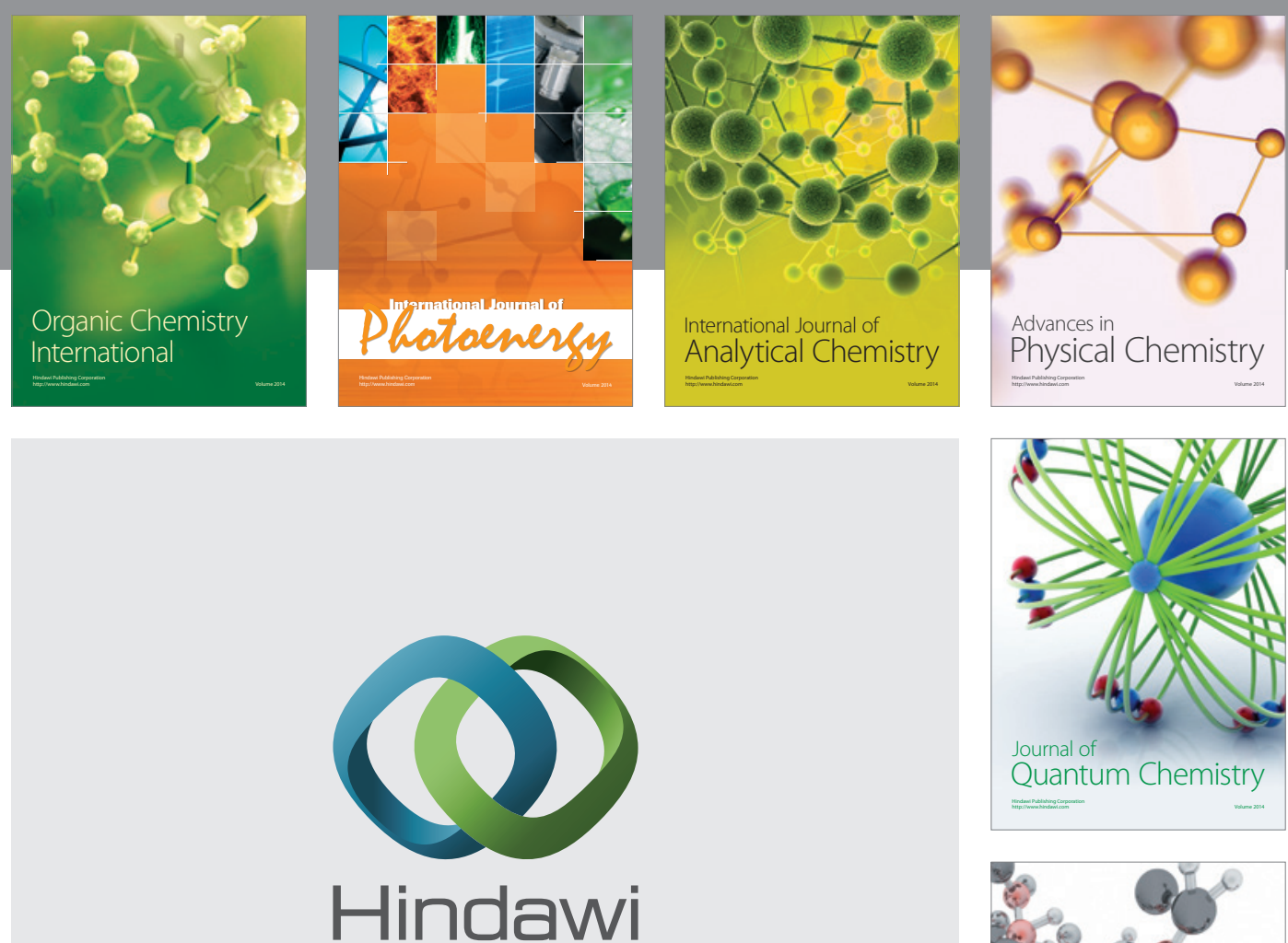

Submit your manuscripts at

http://www.hindawi.com

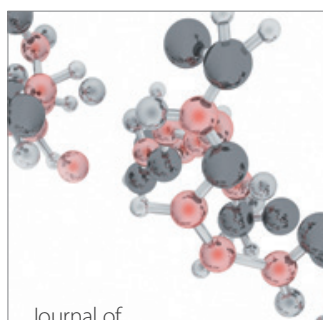

Analytical Methods

in Chemistry

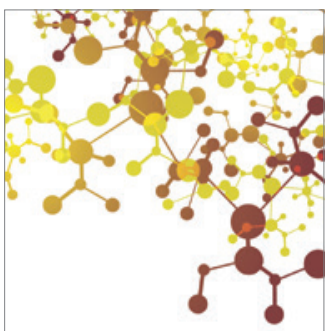

Journal of

Applied Chemistry

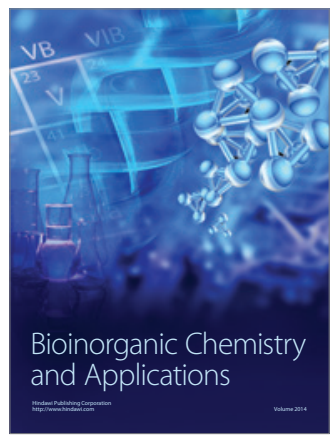

Inorganic Chemistry
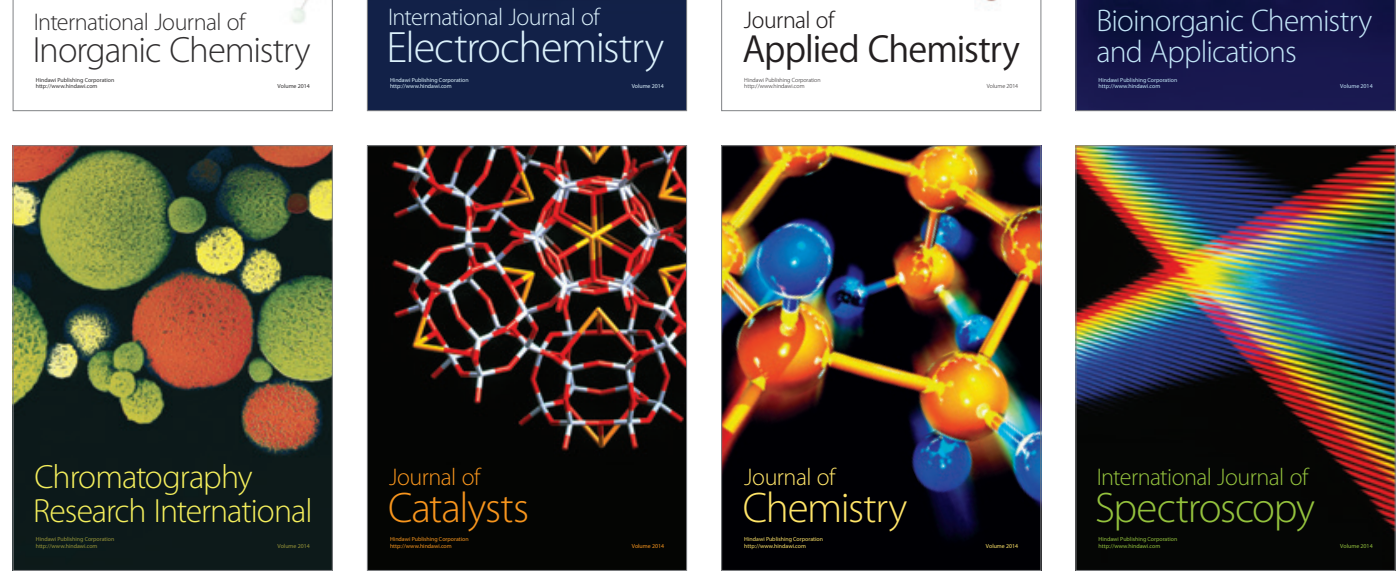